\title{
Reliability of diaphragmatic ultrasonography to detect diaphragm dysfunction in critically ill patients
}

\author{
M Dres ${ }^{1,2^{*}}$, B-P Dubé ${ }^{1}$, J Mayaux ${ }^{1}$, J Delemazure ${ }^{1}$, H Prodanovic $^{1}$, T Similowski ${ }^{1,2}$, A Demoule ${ }^{1,2}$ \\ From ESICM LIVES 2015 \\ Berlin, Germany. 3-7 October 2015
}

\section{Introduction}

The gold standard to diagnose diaphragmatic dysfunction (DD) is the measurement of the intra-thoracic depression is response to a bilateral stimulation of the phrenic nerves (Ptr,stim). This technique is costly, requires expertise and is not widely available at the bedside. On the opposite, ultrasonography is easy to perform but has not been compared.

\section{Objectives}

To validate diaphragm ultrasonography as a tool to detect diaphragm dysfunction in mechanically ventilated patients. Ptr,stim was used as the gold standard.

\section{Methods}

This monocentric prospective study was conducted in a 16 -beds medical ICU. DD was assessed by two independent observers at three key time points:

1) during the first $24 \mathrm{~h}$ of controlled ventilation (CV),

2) as soon as patients could tolerate pressure support ventilation (PSV) and

3) the day of the first spontaneous breathing trial (SBT). Ptr,stim was measured in response to bilateral anterior magnetic stimulation of the phrenic nerves. Mmode ultrasonography was used measure

1) the muscle thickening fraction (TF, (defined as the difference between inspiratory and expiratory muscle thickness divided by the expiratory thickness) of the right hemidiaphragm,

2) the maximal right diaphragmatic excursion was also recorded. A Ptr, stim $<-11 \mathrm{cmH}_{2} \mathrm{O}$ defined DD.

${ }^{1}$ Hopital Pitie Salpetriere, Assistance Publique Hôpitaux de Paris, Respiratory and Critical Care Department, Paris, France

Full list of author information is available at the end of the article

\section{Results}

Seventy-three critically ill patients were investigated (136 measurements: 54 under CV, 32 under PSV and 40 the day of the SBT). DD was present in $79 \%, 85 \%$ and $60 \%$ of the patients under CV, PSV and SBT respectively. Taken together, the measurements performed under PSV and the day of the SBT showed a significant correlation between Ptr, stim and TFd $\left(\mathrm{R}^{2}=0.79, \mathrm{p}<0.001\right)$. A TF $<29 \%$ had a sensitivity and specificity of $85 \%$ and $95 \%$ to detect DD (area under the receiver operating characteristics curve 0.93).

There were no significant correlation between Ptr,stim and

1) diaphragm thickness

2) the maximal excursion of the diaphragm.

\section{Conclusions}

Diaphragm ultrasonography is a reliable method to identify DD in mechanically ventilated patients with an active inspiration. A diaphragm $\mathrm{TF}<29 \%$ is a strong predictor of DD.

\section{Grants}

Martin DRES was supported by Assistance Publique Hôpitaux de Paris.

\section{Authors' details}

${ }^{1}$ Hopital Pitie Salpetriere, Assistance Publique Hôpitaux de Paris, Respiratory and Critical Care Department, Paris, France. 'Sorbonne Universités, UPMC Université Paris 06, INSERM, UMRS 1158 Neurophysiologie Respiratoire Expérimentale et Clinique, Paris, France.

Published: 1 October 2015

doi:10.1186/2197-425X-3-S1-A452

Cite this article as: Dres et al.: Reliability of diaphragmatic

ultrasonography to detect diaphragm dysfunction in critically ill patients. Intensive Care Medicine Experimental 2015 3(Suppl 1):A452.

\section{SpringerOpen ${ }^{\circ}$}

C 2015 Dres et al.; This is an Open Access article distributed under the terms of the Creative Commons Attribution License (http:// creativecommons.org/licenses/by/4.0), which permits unrestricted use, distribution, and reproduction in any medium, provided the original work is properly cited. 